\title{
Intuitionistic Fuzzy Dot d-ideals of d-algebras
}

\author{
Saidur R. Barbhuiya ${ }^{1}$, D. K. Basnet ${ }^{2}$ \\ ${ }^{1}$ Department of Mathematics, Srikishan Sarda College, Hailakandi Assam-788151, India \\ ${ }^{2}$ Department of Mathematical Sciences, Tezpur University, Tezpur Assam-784 028, India
}

Received: 3 July 2015, Revised: 17 August 2015, Accepted: 15 October 2015

Published online: 12 February 2016.

\begin{abstract}
The concept of intuitionistic fuzzy dot d-ideals in d-algebra is introduced. Intuitionistic fuzzy dot subalgebra of d-algebra is defined. Some important results in respect of intuitionistic fuzzy d-ideal, intuitionistic fuzzy dot d-ideals are derived. Effect of Attanasov's operators $\square, \diamond$ and $F_{\alpha, \beta}$ on intuitionistic fuzzy dot d-ideals are studied. Some properties of intuitionistic fuzzy dot d-ideals under homomorphism are investigated.
\end{abstract}

Keywords: d-algebra, fuzzy d-ideal, fuzzy dot d-ideal, intuitionistic fuzzy dot subalgebra, intuitionistic fuzzy dot d-ideal.

\section{Introduction}

The notion of fuzzy subset of a set was introduced by Zadeh [12] in 1965, since then researchers have been engrossed in extending the concepts and results of every concept of mathematics to boarder framework of fuzzy setting. Imai and Iseki [6] introduced BCK-algebras as a generalization of notion of the concept of set theoretic difference and propositional calculus and in the same year Iseki [7] introduced the notion of BCI-algebra which is a generalization of BCK-algebra. $\mathrm{Xi}[10]$ applied the concept of fuzzy set to BCK-algebra and introduced fuzzy subalgebra and fuzzy ideals in BCKalgebra. In 1996, Neggers and Kim [9] introduced the class of d-algebras which is a generalisation of BCK-algebras and investigated relation between d-algebras and BCK-algebras. Akram and Dar[1] introduced the concepts fuzzy d-algebra, fuzzy subalgebra and fuzzy d-ideals of d-algebra. The notion of a fuzzy dot subalgebra of d-algebra were introduced in [8] by Kim. Al-Shehrie[2] introduced the notion of fuzzy dot d-ideals of a d-algebra. Yun et al. [11] applied the concept of intuitionistic fuzzy set to d-algebra and defined intuitionistic fuzzy d-algebra and intuitionistic fuzzy topological dalgebra. Here in this paper, we introduced the notion of intuitionistic fuzzy dot subalgebra and intuitionistic fuzzy dot d-ideals of d-algebra and we investigated several interesting properties.

\section{Preliminaries}

Definition 1. [2] A d-algebra is a non-empty set $X$ with a constant $O$ and a binary operation $*$ satisfying the following axioms:

(i) $x * x=0$

(ii) $0 * x=0$

(iii) $x * y=0$ and $y * x=0 \Rightarrow x=y$ for all $x, y \in X$.

For brevity we also call X a d-algebra. 
Definition 2. [2] A non-empty subset $S$ of a d-algebra $X$ is called a subalgebra of $X$ if $x * y \in S$, for all $x, y \in S$.

Definition 3. [2] A nonempty subset I of a d-algebra $X$ is called an ideal of $X$ if

(i) $0 \in I$

(ii) $x * y \in I$ and $y \in I \Rightarrow x \in I$

(iii) $x \in I$ and $y \in X \Rightarrow x * y \in I$.

Definition 4. [2] A fuzzy subset $\mu$ of $X$ is called a fuzzy dot subalgebra of a d-algebra $X$ if for all $x, y \in X, \mu(x * y) \geq \mu(x) . \mu(y)$, where . (dot) denotes ordinary multiplication.

Definition 5. [2] A fuzzy subset $\mu$ of $X$ is called a fuzzy d-ideal of $X$ if it satisfies the following conditions:

(i) $\mu(0) \geq \mu(x)$

(ii) $\mu(x) \geq \min \{\mu(x * y), \mu(y)\}$

(iii) $\mu(x * y) \geq \min \{\mu(x), \mu(y)\}$.

Definition 6. [2] A fuzzy subset $\mu$ of $X$ is called a fuzzy dot d-ideal of X if it satisfies the following conditions:

(i) $\mu(0) \geq \mu(x)$

(ii) $\mu(x) \geq \mu(x * y) \cdot \mu(y)$

(iii) $\mu(x * y) \geq \mu(x) \cdot \mu(y)$ for all $x, y \in X$.

Definition 7. An intuitionistic fuzzy set (IFS) A of a d-algebra $X$ is an object of the form $\left.A=\left\{<x, \mu_{A}(x), v_{A}(x)\right\rangle \mid x \in X\right\}$, where $\mu_{A}: X \rightarrow[0,1]$ and $v_{A}: X \rightarrow[0,1]$ with the condition $0 \leq \mu_{A}(x)+v_{A}(x) \leq 1, \forall x \in X$. The numbers $\mu_{A}(x)$ and $v_{A}(x)$ denote respectively the degree of membership and the degree of non-membership of the element $x$ in set A. For the sake of simplicity, we shall use the symbol $A=\left(\mu_{A}, v_{A}\right)$ for the intuitionistic fuzzy set $\left.A=\left\{<x, \mu_{A}(x), v_{A}(x)\right\rangle \mid x \in X\right\}$. The function $\pi_{A}(x)=1-\mu_{A}(x)-v_{A}(x)$ for all $x \in X$. Here $\pi_{A}(x)$ is called the degree of hesitance of $x \in A$.

Definition 8. If $A=\left\{<x, \mu_{A}(x), v_{A}(x)>\mid x \in X\right\}$ and $B=\left\{<x, \mu_{B}(x), v_{B}(x)>\mid x \in X\right\}$ are any two IFS of a set $X$, then

$A \subseteq B$ if and only if for all $x \in X, \mu_{A}(x) \leq \mu_{B}(x)$ and $v_{A}(x) \geq v_{B}(x)$,

$A=B$ if and only if for all $x \in X, \mu_{A}(x)=\mu_{B}(x)$ and $v_{A}(x)=v_{B}(x)$,

$A \cap B=\left\{<x,\left(\mu_{A} \cap \mu_{B}\right)(x),\left(v_{A} \cup v_{B}\right)(x)>\mid x \in X\right\}$,

where $\left(\mu_{A} \cap \mu_{B}\right)(x)=\min \left\{\mu_{A}(x), \mu_{B}(x)\right\}$ and $\left(v_{A} \cup v_{B}\right)(x)=\max \left\{v_{A}(x), v_{B}(x)\right\}$,

$A \cup B=\left\{<x,\left(\mu_{A} \cup \mu_{B}\right)(x),\left(v_{A} \cap v_{B}\right)(x)>\mid x \in X\right\}$,

where $\left(\mu_{A} \cup \mu_{B}\right)(x)=\max \left\{\mu_{A}(x), \mu_{B}(x)\right\}$ and $\left(v_{A} \cap v_{B}\right)(x)=\min \left\{v_{A}(x), v_{B}(x)\right\}$.

Definition 9. [2] An intuitionistic fuzzy set $A=\left\{\left\langle x, \mu_{A}(x), v_{A}(x)\right\rangle \mid x \in X\right\}$ of d-algebra $X$ is called an intuitionistic fuzzy subalgebra of $X$ if it satisfies the following conditions:

(i) $\mu_{A}(x * y) \geq \min \left\{\mu_{A}(x), \mu(y)\right\}$

(ii) $v_{A}(x * y) \leq \max \left\{v_{A}(x), v_{A}(y)\right\}$ for all $x, y \in X$.

Definition 10. An intuitionistic fuzzy set $A=\left\{<x, \mu_{A}(x), v_{A}(x)>\mid x \in X\right\}$ of d-algebra $X$ is called an intuitionistic fuzzy $d$-ideal of $X$ if it satisfies the following conditions:

(i) $\mu_{A}(0) \geq \mu_{A}(x)$

(ii) $\mu_{A}(x) \geq \min \left\{\mu_{A}(x * y), \mu(y)\right\}$

(iii) $\mu_{A}(x * y) \geq \min \left\{\mu_{A}(x), \mu_{A}(y)\right\}$ for all $x, y \in X$. 
(iv) $v_{A}(0) \leq v_{A}(x)$

(v) $v_{A}(x) \leq \max \left\{v_{A}(x * y), \mu(y)\right\}$

(vi) $v_{A}(x * y) \leq \max \left\{v_{A}(x), v_{A}(y)\right\}$ for all $x, y \in X$.

Definition 11. For any IFS $A=\left\{<x, \mu_{A}(x), v_{A}(x)>\mid x \in X\right\}$ of $X$ and $\alpha \in[01]$, the operator $\square: \operatorname{IFS}(X) \rightarrow \operatorname{IFS}(X), \diamond:$ $\operatorname{IFS}(X) \rightarrow \operatorname{IFS}(X), D_{\alpha}: \operatorname{IFS}(X) \rightarrow \operatorname{IFS}(X)$ are defined as

1. $\square(A)=\left\{<x, \mu_{A}(x), 1-\mu_{A}(x)>\mid x \in X\right\}$ is called necessity operator

2. $\diamond(A)=\left\{<x, 1-v_{A}(x), v_{A}(x)>\mid x \in X\right\}$ is called possibility operator

3. $D_{\alpha}(A)=\left\{<x, \mu_{A}(x)+\alpha \pi_{A}(x), v_{A}(x)+(1-\alpha) \pi_{A}(x)>\mid x \in X\right\}$ is called $\alpha$-Model operator.

Clearly $\square(A) \subseteq A \subseteq \diamond(A)$ and the equality hold, when $A$ is a fuzzy set also $D_{0}(A)=\square(A)$ and $D_{1}(A)=\diamond(A)$. Therefore the $\alpha$-Model operator $D_{\alpha}(A)$ is an extension of necessity operator $\square(A)$ and possibility operator $\diamond(A)$.

Definition 12. For any IFS $A=\left\{\left\langle x, \mu_{A}(x), v_{A}(x)>\right| x \in X\right\}$ of $X$ and for any $\alpha, \beta \in[01]$ such that $\alpha+\beta \leq 1$, the $(\alpha, \beta)$ model operator $F_{\alpha, \beta}: \operatorname{IFS}(X) \rightarrow \operatorname{IFS}(X)$ is defined as $\left.F_{\alpha, \beta}(A)=\left\{<x, \mu_{A}(x)+\alpha \pi_{A}(x), v_{A}(x)+\beta \pi_{A}(x)\right\rangle \mid x \in X\right\}$, where $\pi_{A}(x)=1-\mu_{A}(x)-v_{A}(x)$ for all $x \in X$. Therefore we can write

$F_{\alpha, \beta}(A)$ as $F_{\alpha, \beta}(A)(x)=\left(\mu_{F_{\alpha, \beta}(A)}(x), v_{F_{\alpha, \beta}(A)}(x)\right)$

where $\mu_{F_{\alpha, \beta}}(x)=\mu_{A}(x)+\alpha \pi_{A}(x)$ and $\left.v_{F_{\alpha, \beta}(A)}(x)\right)=v_{A}(x)+\beta \pi_{A}(x)$.

Clearly, $F_{0,1}(A)=\square(A), F_{1,0}(A)=\diamond(A)$ and $F_{\alpha, 1-\alpha}(A)=D_{\alpha}(A)$.

Remark. If $\mathrm{X}$ and $\mathrm{Y}$ be two d-algebras, then $X \times X$ is also a d-algebra under the binary operation ' ${ }^{\prime}$ defined in $X \times X$ by $(x, y) *(p, q)=(x * p, y * q)$ for all $(x, y),(p, q) \in X \times X$.

Definition 13. Let $X$ and $Y$ be two non empty sets and $f: X \longrightarrow Y$ be a mapping. Let $A$ and $B$ be IFS's of $X$ and $Y$ respectively. Then the image of $A$ under the map $f$ is denoted by $f(A)$ and is defined by $f(A) y=\left(\mu_{f(A)} y, v_{f(A)} y\right)$, where $\mu_{f(A)}(y)=\left\{\begin{array}{l}\vee\left\{\mu_{A}(x): x \in f^{-1}(y)\right\} \\ 0 \quad \text { otherwise }\end{array} \quad v_{f(A)}(y)=\left\{\begin{array}{l}\wedge\left\{v_{A}(x): x \in f^{-1}(y)\right\} \\ 1 \text { otherwise }\end{array}\right.\right.$ also pre image of $B$ under $f$ is denoted by $f^{-1}(B)$ and is defined as

$$
f^{-1}(B)(x)=\left(\mu_{f^{-1}(B)}(x), v_{f^{-1}(B)}(x)\right)=\left(\mu_{B}(f(x)), v_{B}(f(x))\right) ; \forall x \in X .
$$

Remark: Note that $\mu_{A}(x) \leq \mu_{f(A)}(f(x))$ and $v_{A}(x) \geq v_{f(A)}(f(x)) \quad \forall x \in X$ however equality hold when the map $f$ is bijective.

Definition 14. Let $A=<\mu_{A}, v_{A}>$ be intuitionistic fuzzy subset of $X$ and $\alpha, \beta \in[0,1]$ then $(\alpha, \beta)$ cut set of $A$ is $A_{(\alpha, \beta)}=\left\{x \mid x \in X, \mu_{A}(x) \geq \alpha \quad\right.$ and $\left.\quad v_{A}(x) \leq \beta\right\}$

Lemma 1. If $a, b, c, d \in[0,1]$, then

$$
\begin{aligned}
& -\min \{a, b\} \geq a . b \\
& -\max \{a, b\} \leq a+b-a . b \\
& -\min \{a \cdot b, c . d\} \geq \min \{a, c\} . \min \{b, d\} .
\end{aligned}
$$

\section{Intuitionistic fuzzy dot d-ideals of d-algebras}

Definition 15. An intuitionistic fuzzy set $A=\left\{\left\langle x, \mu_{A}(x), v_{A}(x)>\right| x \in X\right\}$ of d-algebra $X$ is called an intuitionistic fuzzy dot subalgebra of $X$ if it satisfies the following conditions: 
(i) $\mu_{A}(x * y) \geq \mu_{A}(x) \cdot \mu(y)$

(ii) $v_{A}(x * y) \leq v_{A}(x)+v_{A}(y)-v_{A}(x) \cdot v_{A}(y)$ for all $x, y \in X$.

Definition 16. An intuitionistic fuzzy set $A=\left\{<x, \mu_{A}(x), v_{A}(x)>\mid x \in X\right\}$ of d-algebra $X$ is called an intuitionistic fuzzy dot $d$-ideal of $X$ if it satisfies the following conditions:

(i) $\mu_{A}(0) \geq \mu_{A}(x)$

(ii) $\mu_{A}(x) \geq \mu_{A}(x * y) \cdot \mu(y)$

(iii) $\mu_{A}(x * y) \geq \mu_{A}(x) \cdot \mu_{A}(y)$ for all $x, y \in X$.

(iv) $v_{A}(0) \leq v_{A}(x)$

(v) $v_{A}(x) \leq v_{A}(x * y)+v_{A}(y)-v_{A}(x * y) \cdot v_{A}(y)$

(vi) $v_{A}(x * y) \leq v_{A}(x)+v_{A}(y)-v_{A}(x) . v_{A}(y)$ for all $x, y \in X$.

Example 1. Consider d-algebra $X=\{0, a, b, c\}$ with the following cayley table.

\begin{tabular}{|c|c|c|c|c|}
\hline$*$ & 0 & $\mathrm{a}$ & $\mathrm{b}$ & $\mathrm{c}$ \\
\hline 0 & 0 & 0 & 0 & 0 \\
\hline $\mathrm{a}$ & $\mathrm{a}$ & 0 & 0 & $\mathrm{a}$ \\
\hline $\mathrm{b}$ & $\mathrm{b}$ & $\mathrm{b}$ & 0 & 0 \\
\hline $\mathrm{c}$ & $\mathrm{c}$ & $\mathrm{c}$ & $\mathrm{a}$ & 0 \\
\hline
\end{tabular}

The intuitionistic fuzzy subset $A=\left\{<x, \mu_{A}(x), v_{A}(x)>\mid x \in X\right\}$ given by $\mu_{A}(0)=\mu_{A}(b)=0.6, \mu_{A}(a)=\mu_{A}(c)=0.5$ and $v_{A}(0)=v_{A}(a)=0.3, v_{A}(b)=v_{A}(c)=0.4$ then it is easy to verify that $A=\left\{\left\langle\mu_{A}(x), v_{A}(x)\right\rangle\right\}$ is an intuitionistic fuzzy dot d-ideal of $X$.

Theorem 1. Every intuitionistic fuzzy d-ideal of d algebra $X$ is an intuitionistic fuzzy dot d-ideal of X.

Proof. Let $A=\left\{\left\langle x, \mu_{A}(x), v_{A}(x)>\right| x \in X\right\}$ be an intuitionistic fuzzy d-ideal of $\mathrm{X}$, therefore we have

(i) $\mu_{A}(0) \geq \mu_{A}(x)$

(ii) $\mu_{A}(x) \geq \min \left\{\mu_{A}(x * y), \mu_{A}(y)\right\}$

(iii) $\mu_{A}(x * y) \geq \min \left\{\mu_{A}(x), \mu_{A}(y)\right\}$ for all $x, y \in X$.

(iv) $v_{A}(0) \leq v_{A}(x)$

(v) $v_{A}(x) \leq \max \left\{v_{A}(x * y), \mu(y)\right\}$

(vi) $v_{A}(x * y) \leq \max \left\{v_{A}(x), v_{A}(y)\right\}$ for all $x, y \in X$.

Now $\mu_{A}(x) \geq \min \left\{\mu_{A}(x * y), \mu(y)\right\} \geq \mu_{A}(x * y) . \mu(y)$ and $\mu_{A}(x * y) \geq \min \left\{\mu_{A}(x) \cdot \mu_{A}(y)\right\} \geq \mu_{A}(x)$. $\mu_{A}(y)$ for all $x, y \in X$. Also $v_{A}(x) \leq \max \left\{v_{A}(x * y), \mu(y)\right\} \leq v_{A}(x * y)+\mu_{A}(y)-v_{A}(x * y) \cdot v_{A}(y)$ and $v_{A}(x * y) \leq \max \left\{v_{A}(x), v_{A}(y)\right\} \leq v_{A}(x)+$ $\mu_{A}(y)-v_{A}(x) \cdot v_{A}(y)$ for all $x, y \in X$.

Hence the proof.

Remark. The converse of Theorem 1 is not true as shown in following Example.

Example 2. Consider d-algebra $X=\{0, a, b\}$ with the following cayley table.

\begin{tabular}{|c|c|c|c|}
\hline$*$ & 0 & $\mathrm{a}$ & $\mathrm{b}$ \\
\hline 0 & 0 & 0 & 0 \\
\hline $\mathrm{a}$ & $\mathrm{b}$ & 0 & $\mathrm{~b}$ \\
\hline $\mathrm{b}$ & $\mathrm{a}$ & $\mathrm{a}$ & 0 \\
\hline
\end{tabular}


The intuitionistic fuzzy subset $A=\left\{<x, \mu_{A}(x), v_{A}(x)>\mid x \in X\right\}$ given by $\mu_{A}(0)=0.6, \mu_{A}(a)=0.4, \mu_{A}(b)=0.5$ and $v_{A}(0)=0.4, v_{A}(a)=0.6, v_{A}(b)=0.5$ then $A=<\mu_{A}, v_{A}>$ is an IF $\operatorname{dot} d$-ideal of $X$. But $A=<\mu_{A}, v_{A}>$ is not an IF $d$-ideal of $X$. Since $\mu_{A}(a)=0.4 \nsupseteq \min \left\{\mu_{A}(a * b), \mu_{A}(b)\right\}=\min \left\{\mu_{A}(b), \mu_{A}(b)\right\}=0.5$.

Theorem 2. Every intuitionistic fuzzy dot d-ideal of a d algebra X is an intuitionistic fuzzy dot subalgebra of X.

Remark. The converse of Theorem 2 is not true as shown in following Example.

Example 3. Consider d-algebra $X$ as in Example 2 and intuitionistic fuzzy subset $A=\left\{\left\langle x, \mu_{A}(x), v_{A}(x)\right\rangle \mid x \in X\right\}$ given by $\mu_{A}(0)=0.3, \mu_{A}(a)=0.5, \mu_{A}(b)=0.4$ and $v_{A}(0)=0.4, v_{A}(a)=0.5, v_{A}(b)=0.3$ then it can be easily verified that $A=<\mu_{A}, v_{A}>$ is an IF dot subalgebra of $X$. But $A=<\mu_{A}, v_{A}>$ is not an IF dot d ideal $X$. Since $\mu_{A}(0)=0.3 \nsupseteq \mu_{A}(a)=$ 0.5 and $v_{A}(0)=0.4 \not \leq \mu_{A}(b)=0.3$.

Lemma 2. If $a, b, p, q \in[01]$, then

$\max (a+b-a \cdot b, p+q-p \cdot q<\max (a, p)+\max (b, q)-\max (a, p) \cdot \max (b, q)<1$ and the equality holds only when $a=$ $b=p=q=1$.

Theorem 3. If $A=\left\{<x, \mu_{A}(x), v_{A}(x)>\mid x \in X\right\}$ and $B=\left\{<x, \mu_{B}(x), v_{B}(x)>\mid x \in X\right\}$ are two intuitionistic fuzzy dot $d$-ideals of $X d$-algebra $X$, Then $(A \cap B)$ is also an intuitionistic fuzzy dot $d$-ideal of $X$.

Proof. We have $A \cap B=\left\{<x,\left(\mu_{A} \cap \mu_{B}\right)(x),\left(v_{A} \cup v_{B}\right)(x)>\mid x \in X\right\}$, where $\left(\mu_{A} \cap \mu_{B}\right)(x)=\min \left\{\mu_{A}(x), \mu_{B}(x)\right\}$ and $\left(v_{A} \cup v_{B}\right)(x)=\max \left\{v_{A}(x), v_{B}(x)\right\}$

Let $x, y \in X$, then

$$
\begin{aligned}
(\mathrm{i})\left(\mu_{A} \cap \mu_{B}\right)(0) & =\min \left\{\mu_{A}(0), \mu_{B}(0)\right\} \\
& =\min \left\{\mu_{A}(0), \mu_{B}(0)\right\} \\
& \geq \min \left\{\mu_{A}(x), \mu_{B}(x)\right\} \\
& =\left(\mu_{A} \cap \mu_{B}\right)(x) \\
\Rightarrow\left(\mu_{A} \cap \mu_{B}\right)(0) & \geq\left(\mu_{A} \cap \mu_{B}\right)(x) \\
(\text { ii })\left(v_{A} \cup v_{B}\right)(0) & =\max \left\{v_{A}(0), v_{B}(0)\right\} \\
& =\max \left\{v_{A}(0), v_{B}(0)\right\} \\
& \leq \max \left\{v_{A}(x), v_{B}(x)\right\} \\
& =\left(v_{A} \cup v_{B}\right)(x) \\
\Rightarrow\left(v_{A} \cup v_{B}\right)(0) & \leq\left(v_{A} \cup v_{B}\right)(x) \\
(\text { iii })\left(\mu_{A} \cap \mu_{B}\right)(x) & =\min \left\{\mu_{A}(x), \mu_{B}(x)\right\} \\
& \geq \min \left\{\mu_{A}(x * y) \cdot \mu_{A}(y), \mu_{B}(x * y) \cdot \mu_{B}(y)\right\} \\
& \left.\geq \min \left\{\mu_{A}(x * y), \mu_{B}(x * y)\right\} \cdot \min \left\{\mu_{A}(y), \mu_{B}(y)\right\}\right\} \\
& \left.\left.=\left(\mu_{A} \cap \mu_{B}\right)(x * y) \cdot\left(\mu_{A} \cap \mu_{B}\right)(y)\right\}\right\} \\
\Rightarrow\left(\mu_{A} \cap \mu_{B}\right)(x) & \left.\left.\geq\left(\mu_{A} \cap \mu_{B}\right)(x * y) \cdot\left(\mu_{A} \cap \mu_{B}\right)(y)\right\}\right\} .
\end{aligned}
$$


(iv) $\left(v_{A} \cup v_{B}\right)(x)$

$=\max \left\{v_{A}(x), v_{B}(x)\right\}$

$\leq \max \left\{v_{A}(x * y)+v_{A}(y)-v_{A}(x * y) \cdot v_{A}(y), v_{B}(x * y)+v_{B}(y)-v_{B}(x * y) \cdot v_{B}(y)\right\}$

$\leq \max \left\{v_{A}(x * y), v_{B}(x * y)\right\}+\max \left\{v_{A}(y), v_{B}(y)\right\}-\max \left\{v_{A}(x * y), v_{B}(x * y)\right\} \cdot \max \left\{v_{A}(y), v_{B}(y)\right\} . \quad$ By Lemma 2

$=\left(v_{A} \cup v_{B}\right)(x * y)+\left(v_{A} \cup v_{B}\right)(y)-\left(v_{A} \cup v_{B}\right)(x * y) \cdot\left(v_{A} \cup v_{B}\right)(y)$.

$\Rightarrow\left(v_{A} \cup v_{B}\right)(x) \leq\left(v_{A} \cup v_{B}\right)(x * y)+\left(v_{A} \cup v_{B}\right)(y)-\left(v_{A} \cup v_{B}\right)(x * y) .\left(v_{A} \cup v_{B}\right)(y)$.

$(\mathrm{v})\left(\mu_{A} \cap \mu_{B}\right)(x * y)=\min \left\{\mu_{A}(x * y), \mu_{B}(x * y)\right\}$

$\left.\geq \min \left\{\mu_{A}(x) \cdot \mu_{A}(y)\right\}, \mu_{B}(x) \cdot \mu_{B}(y)\right\}$

$\geq \min \left\{\mu_{A}(x), \mu_{B}(x)\right\} \cdot \min \left\{\mu_{A}(y), \mu_{B}(y)\right\}$

$\left.\left.=\left(\mu_{A} \cap \mu_{B}\right)(x)\right\} \cdot\left(\mu_{A} \cup \mu_{B}\right)(y)\right\}$

$\left.\Rightarrow\left(\mu_{A} \cap \mu_{B}\right)(x * y) \geq\left(\mu_{A} \cap \mu_{B}\right)(x) .\left(\mu_{A} \cap \mu_{B}\right)(y)\right\}$.

(vi) $\left(v_{A} \cup v_{B}\right)(x * y)$

$=\max \left\{v_{A}(x * y), v_{B}(x * y)\right\}$

$\leq \max \left\{v_{A}(x)+v_{A}(y)-v_{A}(x) \cdot v_{A}(y), v_{B}(x)+v_{B}(y)-v_{B}(x) \cdot v_{B}(y)\right\}$

$\leq \max \left\{v_{A}(x), v_{B}(x)\right\}+\max \left\{v_{A}(y), v_{B}(y)\right\}-\max \left\{v_{A}(x), v_{B}(x)\right\} \cdot \max \left\{v_{A}(y), v_{B}(y)\right\}$. By Lemma 2

$=\left(v_{A} \cup v_{B}\right)(x)+\left(v_{A} \cup v_{B}\right)(y)-\left(v_{A} \cup v_{B}\right)(x) \cdot\left(v_{A} \cup v_{B}\right)(y)$.

$\Rightarrow\left(v_{A} \cup v_{B}\right)(x * y) \leq\left(v_{A} \cup v_{B}\right)(x)+\left(v_{A} \cup v_{B}\right)(y)-\left(v_{A} \cup v_{B}\right)(x) .\left(v_{A} \cup v_{B}\right)(y)$.

Hence $(A \cap B)$ is also an intuitionistic fuzzy dot d-ideal of $\mathrm{X}$.

Theorem 4. IF $C_{\alpha, \beta}(A)=\left\{x \in X \mid \mu_{A}(x) \geq \alpha, v_{A}(x) \leq \beta\right\}$ is an ideal of $X$, then $A=<\mu_{A}, v_{A}>$ is an intuitionistic fuzzy dot d-ideal of $X$, where $\alpha, \beta \in[0,1]$.

Proof. Let $C_{\alpha, \beta}(A)$ is an ideal. To prove $A=<\mu_{A}, v_{A}>$ is an intuitionistic fuzzy dot d-ideal of X. In view of Theorem 1 it is enough to show that $A=<\mu_{A}, v_{A}>$ is an intuitionistic fuzzy d-ideal of X.

Let $x \in X$ such that $\mu_{A}(x)=\alpha$. Also since $0 \in C_{\alpha, \beta}(A)$.

Therefore $\mu_{A}(0) \geq \alpha=\mu_{A}(x)$.

Let $x * y, y \in X$, such that $\mu_{A}(x * y)=\alpha, \mu_{A}(y)=\gamma$ where $\alpha \leq \gamma$.

Then $(x * y), y \in C_{\alpha, \beta}(A)$. Since $C_{\alpha, \beta}(A)$ is an ideal.

Therefore $x \in C_{\alpha, \beta}(A)$, which implies

$\mu_{A}(x) \geq \alpha=\min \{\alpha, \gamma\}=\min \left\{\mu_{A}(x * y), \mu_{A}(y)\right\}$.

Again let $x, y \in X$ such that $\mu_{A}(x)=\alpha, \mu_{A}(y)=\gamma$ where $\alpha \leq \gamma$ Then $x, y \in C_{\alpha, \beta}(A)$ Since $C_{\alpha, \beta}(A)$ is an ideal Therefore $x * y \in C_{\alpha, \beta}(A)$, which implies

$\mu_{A}(x * y) \geq \alpha=\min \{\alpha, \gamma\}=\min \left\{\mu_{A}(x), \mu_{A}(y)\right\}$.

Similarly we can prove $v_{A}(0) \leq v_{A}(x)$

$v_{A}(x) \leq \max \left\{v_{A}(x * y), v_{A}(y)\right\}$ and

$v_{A}(x * y) \leq \max \left\{v_{A}(x), v_{A}(y)\right\}$. 
Theorem 5. If $A=\left\{\left\langle x, \mu_{A}(x), v_{A}(x)\right\rangle \mid x \in X\right\}$ is an intuitionistic fuzzy dot d-ideals of d-algebra $X$, Then $\square A$ and $\diamond A$ is also an intuitionistic fuzzy dot $d$-ideal of $X$.

Proof. We have $\square A=\left\{<x, \mu_{A}(x), \overline{\mu_{A}}(x)>\mid x \in X\right\}$,

$\diamond A=\left\{<x, \overline{v_{A}}(x), v_{A}(x)>\mid x \in X\right\}$,

To prove $\square A$ is an intuitionistic fuzzy dot d-ideal of $\mathrm{X}$. Let $x, y \in X$, then

$$
\begin{aligned}
\mu_{A}(0) & \geq \mu_{A}(x) \\
\therefore 1-\mu_{A}(0) & \leq 1-\mu_{A}(x) \\
\overline{\mu_{A}}(0) & \leq \overline{\mu_{A}}(x)
\end{aligned}
$$

and,

$$
\begin{aligned}
\mu_{A}(x) & \geq \mu_{A}(x * y) \cdot \mu_{A}(y) \\
\overline{\mu_{A}}(x) & =1-\mu_{A}(x) \\
& \leq 1-\mu_{A}(x * y) \cdot \mu_{A}(y) \\
& =1-\mu_{A}(x * y)+1-\mu_{A}(y)-\left(1-\mu_{A}(x * y)\right) \cdot\left(1-\mu_{A}(y)\right) . \\
& =\overline{\mu_{A}}(x * y)+\overline{\mu_{A}}(y)-\overline{\mu_{A}}(x * y) \cdot \overline{\mu_{A}}(y)
\end{aligned}
$$

Again here,

$$
\begin{aligned}
\mu_{A}(x * y) & \geq \mu_{A}(x) \cdot \mu_{A}(y) \\
\overline{\mu_{A}}(x * y) & =1-\mu_{A}(x * y) \\
& \leq 1-\mu_{A}(x) \cdot \mu_{A}(y) \\
& =1-\mu_{A}(x)+1-\mu_{A}(y)-\left(1-\mu_{A}(x) \cdot\left(1-\mu_{A}(y)\right)\right. \\
& =\overline{\mu_{A}}(x)+\overline{\mu_{A}}(y)-\overline{\mu_{A}}(x) \cdot \overline{\mu_{A}}(y) .
\end{aligned}
$$

To prove $\diamond A$ is an intuitionistic fuzzy dot d-ideal of $\mathrm{X}$. Let $x, y \in X$, then

$$
\begin{aligned}
v_{A}(0) & \leq v_{A}(x) \\
\therefore 1-v_{A}(0) & \geq 1-v_{A}(x) \\
\overline{v_{A}}(0) & \geq \overline{v_{A}}(x) .
\end{aligned}
$$

Here, $\quad v_{A}(x) \leq v_{A}(x * y)+v_{A}(y)-v_{A}(x * y) \cdot v_{A}(y)$

$$
\begin{aligned}
\overline{v_{A}}(x) & =1-v_{A}(x) \\
& \geq 1-v_{A}(x * y)-v_{A}(y)+v_{A}(x * y) \cdot v_{A}(y) \\
& =\left(1-v_{A}(x * y)\right) \cdot\left(1-v_{A}(y)\right) \\
& =\overline{v_{A}}(x * y) \cdot \overline{v_{A}}(y) . \\
v_{A}(x * y) & \leq v_{A}(x)+v_{A}(y)-v_{A}(x) \cdot v_{A}(y) \\
\overline{v_{A}}(x * y) & =1-v_{A}(x * y) \\
& \geq 1-v_{A}(x)-v_{A}(y)+v_{A}(x) \cdot v_{A}(y) \\
& =\left(1-v_{A}(x)\right) \cdot\left(1-v_{A}(y)\right) \\
& =\overline{v_{A}}(x) \cdot \overline{v_{A}}(y) .
\end{aligned}
$$

Again here, $\quad v_{A}(x * y) \leq v_{A}(x)+v_{A}(y)-v_{A}(x) \cdot v_{A}(y)$

Remark. The converse of Theorem 5 is not true as shown in following Example. 
Example 4. Consider d-algebra $X$ as in Example 2 and an intuitionistic fuzzy subset $A=\left\{<x, \mu_{A}(x), v_{A}(x)>\mid x \in X\right\}$ given by $\mu_{A}(0)=0.6, \mu_{A}(a)=0.5, \mu_{A}(b)=0.4$ and $v_{A}(0)=0.2, v_{A}(a)=0.3, v_{A}(b)=0.5$ then it can be easily verified that $A=\left\{<\mu_{A}(x), v_{A}(x) \quad>\right\}$ is not an IF $\operatorname{dot}$ d-ideal of $X \quad$. Since $v_{A}(b)=0.5 \not \leq v_{A}(b * 0)+v_{A}(0)-v_{A}(b * 0) \cdot v_{A}(0)=v_{A}(a)+v_{A}(0)-v_{A}(a) \cdot v_{A}(0)=0.2+0.3-0.06=0.44$. But $\square A=\left\{<\mu_{A}(x), \overline{\mu_{A}}(x)>\right\}$ is given by $\mu_{A}(0)=0.6, \mu_{A}(a)=0.5, \mu_{A}(b)=0.4$ and $\overline{\mu_{A}}(0)=0.4, \overline{\mu_{A}}(a)=0.5, \overline{\mu_{A}}(b)=0.6$ is an IF dot d ideal $X$.

Again consider an intuitionistic fuzzy subset $A=\left\{<x, \mu_{A}(x), v_{A}(x)>\mid x \in X\right\}$ given by $\mu_{A}(0)=0.6, \mu_{A}(a)=0.5, \mu_{A}(b)=0.1$ and $v_{A}(0)=0.4, v_{A}(a)=0.5, v_{A}(b)=0.6$ then it can be easily verified that $A=\left\{<\mu_{A}(x), v_{A}(x)>\right\} \quad$ is not an $\quad$ IF $\quad$ dot $\quad$ d-ideal of $X \quad$. Since $\mu_{A}(b)=0.1 \nsupseteq \mu_{A}(b * 0) \cdot \mu_{A}(0)=\mu_{A}(a) \cdot \mu_{A}(0)=0.5 .0 .6=0.3$. But $\diamond A=\left\{<\overline{v_{A}}(x), v_{A}(x)>\right\}$ is given by $\overline{v_{A}}(0)=0.6, \overline{v_{A}}(a)=0.5, \overline{v_{A}}(b)=0.4$ and $v_{A}(0)=0.4, v_{A}(a)=0.5, v_{A}(b)=0.6$ is an IF dot d ideal $X$.

Theorem 6. If $A=<\mu_{A}, v_{A}>$ is an IF d-ideal of d-algebra $X$, then $F_{\alpha, \beta}(A)$ is also an IF $d$ - ideal $X$.

Proof. Let $x \in X$, then $F_{\alpha, \beta}(x)=\left(\mu_{F_{\alpha, \beta}(A)}(x), v_{F_{\alpha, \beta}(A)}(x)\right)$ where $\mu_{F_{\alpha, \beta}(A)}(x)=\mu_{A}(x)+\alpha \pi_{A}(x)$ and $v_{F_{\alpha, \beta}(A)}(x)=v_{A}(x)+$ $\beta \pi_{A}(x)$. Now

$$
\begin{aligned}
\mu_{F_{\alpha, \beta}(A)}(0) & =\mu_{A}(0)+\alpha \pi_{A}(0) \\
& =\mu_{A}(0)+\alpha\left(1-\mu_{A}(0)-v_{A}(0)\right) \\
& =\alpha+(1-\alpha) \mu_{A}(0)-\alpha v_{A}(0) \\
& \geq \alpha+(1-\alpha) \mu_{A}(x)-\alpha v_{A}(x) \\
& =\mu_{A}(x)+\alpha\left(1-\mu_{A}(x)-v_{A}(x)\right) \\
& =\mu_{A}(x)+\alpha \pi_{A}(x)=\mu_{F_{\alpha, \beta}(A)}(x) . \\
\therefore & \mu_{F_{\alpha, \beta}(A)}(0) \geq \mu_{F_{\alpha, \beta}(A)}(x)
\end{aligned}
$$

Similarly we can prove

$$
v_{F_{\alpha, \beta}(A)}(0) \leq v_{F_{\alpha, \beta}(A)}(x) .
$$

$$
\begin{aligned}
& \mu_{F_{\alpha, \beta}(A)}(x) \\
& =\mu_{A}(x)+\alpha \pi_{A}(x) \\
& =\mu_{A}(x)+\alpha\left(1-\mu_{A}(x)-v_{A}(x)\right) \\
& =\alpha+(1-\alpha) \mu_{A}(x)-\alpha v_{A}(x) \\
& \geq \alpha+(1-\alpha) \min \left(\mu_{A}\left((x * y), \mu_{A}(y)\right)-\alpha \max \left(v_{A}\left((x * y), v_{A}(y)\right)\right.\right. \\
& \geq \alpha\left\{1-\max \left(v_{A}\left((x * y), v_{A}(y)\right)\right\}+(1-\alpha) \min \left(\mu_{A}\left((x * y), \mu_{A}(y)\right)\right.\right. \\
& \geq \alpha \min \left(1-v_{A}\left((x * y), 1-v_{A}(y)\right)\right\}+(1-\alpha) \min \left(\mu_{A}\left((x * y), \mu_{A}(y)\right)\right. \\
& \geq \min \left\{\alpha \left(1-v_{A}((x * y))+(1-\alpha) \mu_{A}\left((x * y), \alpha\left(1-v_{A}(y)\right)+(1-\alpha) \mu_{A}(y)\right\}\right.\right. \\
& \geq \min \left\{\mu _ { A } \left((x * y)+\alpha\left(1-\mu_{A}\left((x * y)-v_{A}((x * y)), \mu_{A}(y)+\alpha\left(1-\mu_{A}(y)-v_{A}(y)\right)\right\}\right.\right.\right. \\
& \geq \min \left\{\mu_{F_{\alpha, \beta}(A)}\left((x * y), \mu_{F_{\alpha, \beta}(A)}(y)\right\} .\right.
\end{aligned}
$$




$$
\therefore \mu_{F_{\alpha, \beta}(A)}(x) \geq \min \left\{\mu_{F_{\alpha, \beta}(A)}\left((x * y), \mu_{F_{\alpha, \beta}(A)}(y)\right\}\right.
$$

Similarly we can prove

$$
v_{F_{\alpha, \beta}(A)}(x) \leq \max \left\{v_{F_{\alpha, \beta}(A)}\left((x * y), v_{F_{\alpha, \beta}(A)}(y)\right\} .\right.
$$

$$
\begin{aligned}
& \mu_{F_{\alpha, \beta}(A)}(x * y) \\
& =\mu_{A}(x * y)+\alpha \pi_{A}(x * y) \\
& =\mu_{A}(x * y)+\alpha\left(1-\mu_{A}(x * y)-v_{A}(x * y)\right) \\
& =\alpha+(1-\alpha) \mu_{A}(x * y)-\alpha v_{A}(x * y) \\
& \geq \alpha+(1-\alpha) \min \left(\mu_{A}(x), \mu_{A}(y)\right)-\alpha \max \left(v_{A}(x), v_{A}(y)\right) \\
& \geq \alpha\left\{1-\max \left(v_{A}(x), v_{A}(y)\right)\right\}+(1-\alpha) \min \left(\mu_{A}(x), \mu_{A}(y)\right) \\
& \left.\geq \alpha \min \left(1-v_{A}(x), 1-v_{A}(y)\right)\right\}+(1-\alpha) \min \left(\mu_{A}(x), \mu_{A}(y)\right) \\
& \geq \min \left\{\alpha\left(1-v_{A}(x)\right)+(1-\alpha) \mu_{A}(x), \alpha\left(1-v_{A}(y)\right)+(1-\alpha) \mu_{A}(y)\right\} \\
& \geq \min \left\{\mu_{A}(x)+\alpha\left(1-\mu_{A}(x)-v_{A}(x)\right), \mu_{A}(y)+\alpha\left(1-\mu_{A}(y)-v_{A}(y)\right)\right\} \\
& \geq \min \left\{\mu_{F_{\alpha, \beta}(A)}(x), \mu_{F_{\alpha, \beta}(A)}(y)\right\} . \\
& \quad \therefore \mu_{F_{\alpha, \beta}(A)}(x * y) \geq \min \left\{\mu_{F_{\alpha, \beta}(A)}(x), \mu_{F_{\alpha, \beta}(A)}(y)\right\}
\end{aligned}
$$

Similarly we can prove

$$
v_{F_{\alpha, \beta}(A)}(x * y) \leq \max \left\{v_{F_{\alpha, \beta}(A)}(x), v_{F_{\alpha, \beta}(A)}(y)\right\} .
$$

Hence $F_{\alpha, \beta}(A)$ is an IF d-ideal of $B G$-algebra X.

Theorem 7. If $A=<\mu_{A}, v_{A}>$ is an IF d-ideal of d-algebra $X$, then $F_{\alpha, \beta}(A)$ is also an IF dot d-ideal $X$.

Proof. It follows from Theorem 6 and Theorem 1.

Remark. The converse of Theorem 6 is not true as shown in following Example.

Example 5. Consider d-algebra $X$ as in Example 2 and intuitionistic fuzzy subset $A=\left\{\left\langle x, \mu_{A}(x), v_{A}(x)\right\rangle \mid x \in X\right\}$ given by $\mu_{A}(0)=0.55, \mu_{A}(a)=0.6, \mu_{A}(b)=0.5$ and $v_{A}(0)=0.3, v_{A}(a)=0.4, v_{A}(b)=0.35$ then $A$ is not an IF d- ideal of $\mathrm{X}$, since $\mu_{A}(0)=0.55 \nsupseteq \mu_{A}(a)=0.6$. But $\pi_{A}(0)=0.1, \pi_{A}(a)=0.0, \pi_{A}(b)=0.15$, take $\alpha=0.6, \beta=0.3$ then $\mu_{F_{0.6,0.3}(A)}(0)=$

$0.61, \mu_{F_{0.6,0.3}(A)}(a)=0.6, \mu_{F_{0.6,0.3}(A)}(b)=0.59$ and $v_{F_{0.6,0.3}(A)}(0)=0.33, v_{F_{0.6,0.3}(A)}(a)=0.4, v_{F_{0.6,0.3}(A)}(b)=0.395$. Then it is easy to verify that $F_{0.6,0.3}(A)$ is also an IF dot d- ideal X.

\section{Homomorphism of $d$-algebras and intuitionistic fuzzy dot $d$-ideals}

Definition 17. Let $X$ and $X^{\prime}$ be two d-algebras, then a mapping $f: X \rightarrow X^{\prime}$ is said to be homomorphism if $f(x * y)=$ $f(x) * f(y) \forall x, y \in X$.

Theorem 8. Let $X$ and $X^{\prime}$ be two d-algebras and $f: X \rightarrow X^{\prime}$ be a homomorphism. Then $f(0)=0^{\prime}$.

Proof. Let $x \in X$ therefore $f(x) \in X^{\prime}$. Now $f(0)=f(x * x)=f(x) * f(x)=0 * 0=0^{\prime}$.

Theorem 9. Let $f: X \rightarrow X^{\prime}$ be an onto homomorphism of d-algebras, Let A be an intuitionistic fuzzy dot $d$-ideal of $X^{\prime}$, then the pre-image $f^{-1}(A)$ of $A$ under $f$ is an intuitionistic fuzzy dot $d$-ideal of $X$. 
Proof. $f^{-1}(A)$ is defined as

$$
f^{-1}(A)(x)=f^{-1}\left(\mu_{A}, v_{A}\right)(x)=\left(f^{-1}\left(\mu_{A}\right), f^{-1}\left(v_{A}\right)\right)(x)=\left(f^{-1}\left(\mu_{A}\right)(x), f^{-1}\left(v_{A}\right)(x)\right)=\left(\left(\mu_{A}\right) f(x),\left(v_{A}\right) f(x)\right) \forall x \in X .
$$

$$
\begin{aligned}
f^{-1}\left(\mu_{A}\right)(0) & =\mu_{A} f(0) \\
& \geq \mu_{A} f(x) \\
& =f^{-1}\left(\mu_{A}\right)(x) .
\end{aligned}
$$

Therefore, $\quad f^{-1}\left(\mu_{A}\right)(0) \geq f^{-1}\left(\mu_{A}\right)(x)$

$$
\begin{aligned}
f^{-1}\left(\mu_{A}\right)(x) & =\mu_{A} f(x) \\
& \geq \mu_{A}(f(x) * f(y)) \cdot \mu_{A} f(y) \\
& =\mu_{A} f(x * y) \cdot \mu_{A} f(y) \\
& =f^{-1}\left(\mu_{A}\right)(x * y) \cdot f^{-1}\left(\mu_{A}\right)(y) .
\end{aligned}
$$

Therefore, $\quad f^{-1}\left(\mu_{A}\right)(x) \geq f^{-1}\left(\mu_{A}\right)(x * y) \cdot f^{-1}\left(\mu_{A}\right)(y)$

$$
\begin{aligned}
f^{-1}\left(\mu_{A}\right)(x * y) & =\mu_{A} f(x * y) \\
& \geq \mu_{A}(f(x)) \cdot \mu_{A} f(y) \\
& =\mu_{A} f(x) \cdot \mu_{A} f(y) \\
& =f^{-1}\left(\mu_{A}\right)(x) \cdot f^{-1}\left(\mu_{A}\right)(y) .
\end{aligned}
$$

Therefore, $f^{-1}\left(\mu_{A}\right)(x * y) \geq f^{-1}\left(\mu_{A}\right)(x) \cdot f^{-1}\left(\mu_{A}\right)(y)$

Also

$$
\begin{aligned}
f^{-1}\left(v_{A}\right)(0) & =v_{A} f(0) \\
& \leq v_{A} f(x) \\
& =f^{-1}\left(v_{A}\right)(x) .
\end{aligned}
$$

Therefore, $\quad f^{-1}\left(v_{A}\right)(0) \leq f^{-1}\left(v_{A}\right)(x)$

$$
\begin{aligned}
f^{-1}\left(v_{A}\right)(x) & =v_{A} f(x) \\
& \leq v_{A}(f(x) * f(y))+v_{A} f(y)-v_{A}(f(x) * f(y)) \cdot v_{A} f(y) \\
& =v_{A}(f(x * y))+v_{A} f(y)-v_{A}(f(x * y)) \cdot v_{A} f(y) \\
& =f^{-1}\left(v_{A}\right)(x * y)+f^{-1}\left(v_{A}\right)(y)-f^{-1}\left(v_{A}\right)(x * y) \cdot f^{-1}\left(v_{A}\right)(y) .
\end{aligned}
$$

Therefore, $\quad f^{-1}\left(v_{A}\right)(x) \leq f^{-1}\left(v_{A}\right)(x * y)+f^{-1}\left(v_{A}\right)(y)-f^{-1}\left(v_{A}\right)(x * y) \cdot f^{-1}\left(v_{A}\right)(y)$

$$
\begin{aligned}
f^{-1}\left(v_{A}\right)(x * y) & =v_{A} f(x * y) \\
& \leq v_{A} f(x)+v_{A} f(y)-v_{A} f(x) \cdot v_{A} f(y) \\
& =f^{-1}\left(v_{A}\right)(x)+f^{-1}\left(v_{A}\right)(y)-f^{-1}\left(v_{A}\right)(x) \cdot f^{-1}\left(v_{A}\right)(y) \\
\left.f^{-1}\left(v_{A}\right)(x * y)\right) & \leq f^{-1}\left(v_{A}\right)(x)+f^{-1}\left(v_{A}\right)(y)-f^{-1}\left(v_{A}\right)(x) \cdot f^{-1}\left(v_{A}\right)(y) .
\end{aligned}
$$

Hence $f^{-1}(A)$ is an intuitionistic fuzzy dot d-ideal of $\mathrm{X}$.

Theorem 10. An onto homomorphic image of an intuitionistic fuzzy dot d-ideal with the sup \& inf property is an intuitionistic fuzzy dot d-ideal. 
Proof. Let $f: X \rightarrow X^{\prime}$ be an onto homomorphism of d-algebras, $A=\left\{\left\langle x, \mu_{A}(x), v_{A}(x)>\right| x \in X\right\}$ be an intuitionistic fuzzy dot d-ideal of $X$, and $f(A)$ be the image of A under $\mathrm{f}$. To prove $f(A)$ is an intuitionistic fuzzy dot d-ideal of $\mathrm{X}$ '

$$
\begin{aligned}
& f(\mu)\left(0^{\prime}\right)=\sup _{z \in f^{-1}\left(0^{\prime}\right)} \mu(z)=\mu(0) \geq \mu(x) \quad \forall x \in X \\
& \Rightarrow f(\mu)\left(0^{\prime}\right) \geq \sup _{z \in f^{-1}\left(x^{\prime}\right)} \mu(z)=f(\mu)\left(x^{\prime}\right) \quad \forall x^{\prime} \in X^{\prime} .
\end{aligned}
$$

Let $x_{2}, y_{2} \in X^{\prime}$ be any elements, as $\mathrm{f}$ is onto, there exists unique $x_{1}, y_{1} \in X$ such that $f\left(x_{1}\right)=x_{2}, f\left(y_{1}\right)=y_{2}$, and $f\left(x_{1} *\right.$ $\left.y_{1}\right)=f\left(x_{1}\right) * f\left(y_{1}\right)=x_{2} * y_{2}$. Now $f(A)\left(x_{2}\right)=\left(\mu_{f(A)}\left(x_{2}\right), v_{f(A)}\left(x_{2}\right)\right)$. But $\mu_{f(A)}\left(x_{2}\right)=\sup _{z \in f^{-1}\left(x_{2}\right)} \mu_{A}(z)=\mu_{A}(t)$, where $f(t)=x_{2}=f\left(x_{1}\right) \Rightarrow t=x_{1}$.

$$
\begin{aligned}
\mu_{f(A)}\left(x_{2}\right)=\mu_{A}(t) & =\mu_{A}\left(x_{1}\right) \\
& \geq \mu_{A}\left(x_{1} * y_{1}\right) \cdot \mu_{A}\left(y_{1}\right) \\
& \geq \mu_{f(A)} f\left(x_{1} * y_{1}\right) \cdot \mu_{f(A)} f\left(y_{1}\right) \\
& =\mu_{f(A)}\left(x_{2} * y_{2}\right) \cdot \mu_{f(A)} y_{2} . \\
\mu_{f(A)}\left(x_{2} * y_{2}\right) & =\sup _{z \in f^{-1}\left(x_{2} * y_{2}\right)} \mu_{A}(z) \\
& =\mu_{A}(t), \quad \text { where } f(t)=x_{2} * y_{2}=f\left(x_{1}\right) * f\left(y_{1}\right)=f\left(x_{1} * y_{1}\right) \\
& =\mu_{A}\left(x_{1} * y_{1}\right) \\
& \geq \mu_{A}\left(x_{1}\right) \cdot \mu_{A}\left(y_{1}\right) \\
& \geq \mu_{f(A)} f\left(x_{1}\right) \cdot \mu_{f(A)} f\left(y_{1}\right) \\
& =\mu_{f(A)}\left(x_{2}\right) \cdot \mu_{f(A)}\left(y_{2}\right) .
\end{aligned}
$$

$$
\begin{aligned}
f(v)\left(0^{\prime}\right) & =\inf _{z \in f^{-1}\left(0^{\prime}\right)} v(z)=v(0) \leq v(x) \quad \forall x \in X \\
\Rightarrow f(v)\left(0^{\prime}\right) & \leq \inf _{z \in f^{-1}\left(x^{\prime}\right)} v(z)=f(v)\left(x^{\prime}\right) \quad \forall x^{\prime} \in X^{\prime} . \\
v_{f(A)}\left(x_{2}\right) & =\inf _{z \in f^{-1}\left(x_{2}\right)} v_{A}(z) \\
& =v_{A}(t), \quad \text { where } f(t)=x_{2}=f\left(x_{1}\right) \\
& =v_{A}\left(x_{1}\right) \\
& \leq v_{A}\left(x_{1} * y_{1}\right)+v_{A}\left(y_{1}\right)-v_{A}\left(x_{1} * y_{1}\right) \cdot v_{A}\left(y_{1}\right) \\
& \leq v_{f(A)} f\left(x_{1} * y_{1}\right)+v_{f(A)}\left(y_{1}\right)-v_{f(A)}\left(x_{1} * y_{1}\right) \cdot v_{f(A)}\left(y_{1}\right) \\
& =v_{f(A)}\left(x_{2} * y_{2}\right)+v_{f(A)}\left(y_{2}\right)-v_{f(A)}\left(x_{2} * y_{2}\right) \cdot v_{f(A)}\left(y_{1}\right) .
\end{aligned}
$$

$$
\begin{aligned}
v_{f(A)}\left(x_{2} * y_{2}\right) & =\inf _{z \in f^{-1}\left(x_{2} * y_{2}\right)} v_{A}(z) \\
& =v_{A}(t), \quad \text { where } f(t)=x_{2} * y_{2}=f\left(x_{1}\right) * f\left(y_{1}\right)=f\left(x_{1} * y_{1}\right) \\
& =v_{A}\left(x_{1} * y_{1}\right) \\
& \leq v_{A}\left(x_{1}\right)+\mu_{A}\left(y_{1}\right)-v_{A}\left(x_{1}\right) \cdot v_{A}\left(y_{1}\right) \\
& \leq v_{f(A)} f\left(x_{1}\right)+v_{f(A)} f\left(y_{1}\right)-v_{f(A)} f\left(x_{1}\right) \cdot v_{f(A)} f\left(y_{1}\right) \\
& =v_{f(A)}\left(x_{2}\right)+v_{f(A)}\left(y_{2}\right)-v_{f(A)}\left(x_{2}\right) \cdot v_{f(A)} f\left(\left(y_{2}\right)\right) .
\end{aligned}
$$


Hence from above $f(A)$ is an intuitionistic fuzzy dot d-ideal of $X$.

\section{Conclusions}

In this paper, we have studied intuitionistic fuzzy dot d-ideals of $d$-algebras and obtained some interesting results. We have shown that intersection of any two intuitionistic fuzzy dot d-ideals is a intuitionistic fuzzy dot d-ideal. Intuitionistic fuzzy dot d-ideals is invarient under model operators $\square, \diamond$ and $F_{\alpha, \beta}$. By using same idea, we can define intuitionistic fuzzy dot d-ideal in other algebric systems such as BCK/BCI/BG/BF-algebras etc.

\section{Acknowledgements}

The author would like to express his sincere thanks to the referees for their valuable suggestions and helpful comments in improving this paper.

\section{References}

[1] M. Akram and K. H. Dhar, on fuzzy d algebras, Punjab University Journal of Math, 37(2005), 61-76.

[2] N.O. Al-Shehrie, On fuzzy dot d ideals of d algebras, Advances in Algebra (ISSN 0973-6964), 2(2009), 1-8.

[3] S. R. Barbhuiya, $(\in, \in \vee q)$-Intuitionistic Fuzzy Ideals of BCK/BCI-algebra, Notes on Intuitionistic Fuzzy Sets, 21(1),(2015), 2442.

[4] S. R. Barbhuiya, $\left(a, b ; \in_{a}, \in_{a} \vee q_{(a, b)}\right)$-Fuzzy Ideals of BG-Algebra, Asian Journal of Mathematics and Computer Research, 6(4) (2015), 299-311.

[5] S. R. Barbhuiya, $(\in, \in \vee q)$-Fuzzy Ideals of BG-algebras with respect to t-norm, New Trends in Mathematical Sciences, 3(4), (2015), 196-210.

[6] Y. Imai and K. Iseki, On Axiom systems of Propositional calculi 15, Proc. Japan Academy, 42 (1966), 19-22.

[7] K. Iseki, An algebra related with a propositional calculus, Proc. Japan Academy,42 (1966), 26-29.

[8] K. H. Kim, on fuzzy dot subalgebras of d-algebras, International Mathematical Forum, 4(2009), 645-651.

[9] J. Neggers and H .S. Kim, on d-algebras, Math.Slovaca, 49(1996), 19-26.

[10] O. G. Xi, Fuzzy BCK algebras, Math Japonica, 36(1991), 935-942.

[11] Y. B. Yun, H. S. Kim and D. S. Yoon, Intuitionistic fuzzy d-algebras, Scientiae Mathematicae Japonicae Online, e-2006, 12891297.

[12] L. A. Zadeh, Fuzzy sets, Information and Control 8 (1965), 338-353. 